\title{
Spin-orbit interaction strength and anisotropy in III-V semiconductor heterojunctions
}

\author{
M. A. Toloza Sandoval, ${ }^{1}$ A. Ferreira da Silva, ${ }^{1}$ E. A. de Andrada e Silva, ${ }^{2}$ and G. C. La Rocca ${ }^{3}$ \\ ${ }^{1}$ Instituto de Física, Universidade Federal da Bahia 40210-340, Salvador, Bahia, Brazil \\ ${ }^{2}$ Instituto Nacional de Pesquisas Espaciais, Caixa Postale 515, 12201-970 São José dos Campos, São Paulo, Brazil \\ ${ }^{3}$ Scuola Normale Superiore and CNISM, Piazza dei Cavalieri 7, I-56126 Pisa, Italy \\ (Received 25 October 2012; revised manuscript received 27 December 2012; published 13 February 2013)
}

\begin{abstract}
The spin-orbit interaction strength for electrons in III-V semiconductor heterojunctions and the corresponding in-plane anisotropy are theoretically studied, considering Rashba and Dresselhaus contributions. Starting from a variational solution of Kane's effective Hamiltonian for the Rashba-split subbands, the total spin-orbit splitting at the Fermi level of the two-dimensional electron gas in III-V heterojunctions is calculated analytically, as a function of the electron density and wave-vector direction, by adding the Dresselhaus contribution within quasidegenerate first-order perturbation theory. Available GaAs and InGaAs experimental data are discussed. Effects of the barrier penetration are identified, and the spin-orbit anisotropy is shown to be determined by more than one parameter, even in the small- $k$ limit, contrary to the commonly used $\alpha / \beta$ (where $\alpha$ is the Rashba and $\beta$ the Dresselhaus interaction) single-parameter picture.
\end{abstract}

DOI: 10.1103/PhysRevB.87.081304

With the goal of further pushing the limits of data storage and processing devices, research in semiconductor spintronics has been largely based on the Datta-Das spin transistor. ${ }^{1,2}$ The functioning of such an ideal device is based on gate control of the spin precession of the conducting electrons through the Rashba [or structure inversion asymmetry (SIA)] spin-orbit (SO) coupling in semiconductor heterojunctions. However, despite recent and promising progress, ${ }^{3,4}$ we are still far from a real device. In particular, the SO interaction in an active III-V heterojunction is still not well known, especially regarding its in-plane anisotropy, which is mainly due to corrections from the intrinsic or bulk inversion asymmetry (BIA) SO contribution (the Dresselhaus contribution). In this Rapid Communication, an accurate and particularly transparent solution for the spin-orbit splitting in the conducting electron states in III-V semiconductor heterojunctions is presented. It includes both Rashba and Dresselhaus contributions and is shown to be in reasonable agreement with experiment.

This anisotropy is special because it can also be tuned with the gate voltage so as to make, for example, the SO splitting at the Fermi energy negligible for electrons moving along given in-plane directions, suppressing the relaxation of their spins and forming the so-called persistent spin helix modes, as recently observed. ${ }^{5,6}$ Such anisotropy can be seen to be due to the interplay (or interference) between the two contributions mentioned above. For instance, it is known that in III-V heterojunctions grown along the [001] crystallographic direction, the splitting is maximum for electrons traveling along the direction [110] (constructive interference) and minimum along [110] (destructive interference) ${ }^{7-10}$ However, this picture with a simple twofold rotational symmetry (with respect to the direction of $\vec{k}_{\|}$) is exact only in the linear- $k_{\|}$ and infinite-barrier approximation. ${ }^{7}$ In this approximation, the in-plane SO anisotropy is determined by a single parameter, the so-called $\alpha / \beta$ ratio (i.e., the ratio of the Rashba to the Dresselhaus interaction), and the above mentioned zero-splitting situation occurs along [110] when $\alpha / \beta=1$, corresponding to Rashba and Dresselhaus SO terms with the same strength. ${ }^{7}$ However, as shown here, barrier penetration effects as well as higher-order terms in $k_{\|}$cannot be neglected
PACS number(s): 73.20.-r, 75.70.Tj, 73.22.-f

in the determination of the SO in-plane symmetry of actual III-V semiconductor heterojunctions. Including these effects, the total SO splitting in III-V heterojunctions is calculated here in a particularly transparent and accurate way. Specific results for $\mathrm{AlGaAs} / \mathrm{GaAs}$ and InAlAs/InGaAs structures are shown to be in much better agreement with the experimental data than the simplified and commonly used $\alpha / \beta$ parametrization.

We start from a recently proposed spin-resolved variational solution for the Rashba-split electronic subbands. ${ }^{11,12}$ Within the standard envelope function approximation based on the Kane $\vec{k} \cdot \vec{p}$ model for the bulk, the envelope function of such split subbands satisfies

$$
H_{R}\left|\Psi_{\uparrow \downarrow}\right\rangle=\varepsilon_{\uparrow \downarrow}^{R}\left(k_{\|}\right)\left|\Psi_{\uparrow \downarrow}\right\rangle,
$$

where the effective Hamiltonian $H_{R}$ includes penetration and renormalized parameters in the barrier, as well as band nonparabolicity in the well; ${ }^{11,12}$ and $\left|\Psi_{\uparrow \downarrow}\right\rangle=e^{i \vec{k}_{\|} \cdot \vec{r}_{\|}}\left|f_{\uparrow \downarrow}(z)\right\rangle \chi_{\uparrow \downarrow}(\vartheta)$ are the variational envelope functions for the electrons with spins up and down along the direction perpendicular to $\vec{k}_{\|}=\left(k_{\|}, \vartheta\right)$. The spin part

$$
\chi_{\uparrow}(\vartheta)=\left(\begin{array}{l}
\cos (\vartheta / 2) \\
-i \sin (\vartheta / 2)
\end{array}\right), \quad \chi_{\downarrow}(\vartheta)=\left(\begin{array}{l}
-i \sin (\vartheta / 2) \\
\cos (\vartheta / 2)
\end{array}\right)
$$

depends only on the $\vec{k}_{\|}$direction $\vartheta$ (the angle it makes with the $x$ axis; note that for simplicity the spin is quantized along the $y$ direction), while the scalar part $\left|f_{\uparrow \downarrow}(z)\right\rangle$ depends only on its modulus $k_{\|}$. With the interface at $z=0$, the trial functions for such an envelope function read

$$
\left\langle z \mid f_{\uparrow \downarrow}\right\rangle= \begin{cases}A_{\uparrow \downarrow} e^{k_{b} z / 2}, & z \leqslant 0, \\ B_{\uparrow \downarrow}\left(z+c_{\uparrow \downarrow}\right) e^{-b z / 2}, & z \geqslant 0,\end{cases}
$$

where the variational parameter $b$ (determined by minimizing the total energy) and the parameters $A, B$, and $c$ (determined by the boundary and normalization conditions) do not depend on $\vartheta$. The details of such a variational solution are given in Ref. 12. The resulting spin splitting

$$
\Delta_{R}\left(k_{\|}\right)=\left|\varepsilon_{\uparrow}^{R}\left(k_{\|}\right)-\varepsilon_{\downarrow}^{R}\left(k_{\|}\right)\right|
$$


does not depend on the direction of $\vec{k}_{\|}$and represents the usual Rashba splitting, which in the linear approximation is commonly written as $\Delta_{R}=2 \alpha k_{\|} \cdot{ }^{13}$

The effects of the Dresselhaus bulk contribution ${ }^{14}$ are due to remote bands not included in the Kane model. ${ }^{15}$ They can be studied by treating within quasidegenerate firstorder perturbation theory the additional contribution $H_{D}$ to the conduction band effective Hamiltonian, ${ }^{8}$ which is given by

$H_{D}=\gamma\left[\sigma_{x} k_{x}\left(k_{y}^{2}-k_{z}^{2}\right)+\sigma_{y} k_{y}\left(k_{z}^{2}-k_{x}^{2}\right)+\sigma_{z} k_{z}\left(k_{x}^{2}-k_{y}^{2}\right)\right]$,

where $x, y$, and $z$ correspond to the cubic crystallographic directions and $\vec{\sigma}$ to the Pauli matrix vector. The total Hamiltanian $H=H_{R}+H_{D}$ is then written in the basis set formed with the above unperturbed eigenstates, i.e.,

$$
H=\left(\begin{array}{ll}
H_{\uparrow \uparrow} & H_{\uparrow \downarrow} \\
H_{\downarrow \uparrow} & H_{\downarrow \downarrow}
\end{array}\right),
$$

where $H_{\uparrow \uparrow}=\left\langle\Psi_{\uparrow}|H| \Psi_{\uparrow}\right\rangle, H_{\downarrow \downarrow}=\left\langle\Psi_{\downarrow}|H| \Psi_{\downarrow}\right\rangle$, and so on, given by

$H_{\uparrow \uparrow}=\varepsilon_{\uparrow}^{R}\left(k_{\|}\right)+\left\langle\gamma(z) k_{z}^{2}\right\rangle_{\uparrow \uparrow} \sin (2 \vartheta) k_{\|}-\frac{1}{2}\langle\gamma(z)\rangle_{\uparrow \uparrow} \sin (2 \vartheta) k_{\|}^{3}$,

$H_{\downarrow \downarrow}=\varepsilon_{\downarrow}^{R}\left(k_{\|}\right)-\left\langle\gamma(z) k_{z}^{2}\right\rangle_{\downarrow \downarrow} \sin (2 \vartheta) k_{\|}+\frac{1}{2}\langle\gamma(z)\rangle_{\downarrow \downarrow} \sin (2 \vartheta) k_{\|}^{3}$,

and

$$
H_{\uparrow \downarrow}=i\left\langle\gamma(z) k_{z}^{2}\right\rangle_{\uparrow \downarrow} \cos (2 \vartheta) k_{\|}=H_{\downarrow \uparrow}^{*} ;
$$

with $\langle\gamma(z)\rangle_{\uparrow \uparrow}=\left\langle f_{\uparrow}|\gamma(z)| f_{\uparrow}\right\rangle,\left\langle\gamma(z) k_{z}^{2}\right\rangle_{\uparrow \uparrow}=\left\langle f_{\uparrow}\right|(-i d / d z)$ $\gamma(z)(-i d / d z)\left|f_{\uparrow}\right\rangle$, and so on (note that the bulk $\gamma$ parameter varies along the growth direction $z$ and it is then necessary to symmetrize these integrals). ${ }^{8}$ Then, after straightforward diagonalization, we obtain the following perturbed eigenvalues:

$$
\varepsilon_{ \pm}\left(k_{\|}, \vartheta\right)=\bar{\varepsilon}\left(k_{\|}, \vartheta\right) \pm \frac{1}{2} \Delta_{s}\left(k_{\|}, \vartheta\right)
$$

where the spin-independent part reads

$$
\bar{\varepsilon}\left(k_{\|}, \vartheta\right)=\frac{1}{2}\left\{\varepsilon_{\uparrow}^{R}\left(k_{\|}\right)+\varepsilon_{\downarrow}^{R}\left(k_{\|}\right)+\left[\left\langle\gamma(z) k_{z}^{2}\right\rangle_{\downarrow \downarrow}-\left\langle\gamma(z) k_{z}^{2}\right\rangle_{\uparrow \uparrow}+\frac{1}{2}\left[\langle\gamma(z)\rangle_{\uparrow \uparrow}-\langle\gamma(z)\rangle_{\downarrow \downarrow}\right] k_{\|}^{2}\right] k_{\|} \sin 2 \vartheta\right\}
$$

and the SO splitting is given by

$$
\Delta_{s}\left(k_{\|}, \vartheta\right)=\sqrt{\left\{\Delta_{R}\left(k_{\|}\right)-\left[\left\langle\gamma(z) k_{z}^{2}\right\rangle_{\uparrow \uparrow}+\left\langle\gamma(z) k_{z}^{2}\right\rangle_{\downarrow \downarrow}-\frac{1}{2}\left[\langle\gamma(z)\rangle_{\uparrow \uparrow}+\langle\gamma(z)\rangle_{\downarrow \downarrow}\right] k_{\|}^{2}\right] k_{\|} \sin 2 \vartheta\right\}^{2}+4\left\langle\gamma(z) k_{z}^{2}\right\rangle_{\uparrow \downarrow}^{2} k_{\|}^{2} \cos ^{2} 2 \vartheta} .
$$

In the infinite-barrier limit, this expression for the SO splitting exactly reproduces Eq. (19) in Ref. 16, extending it to the general finite-barrier case. Barrier penetration leads to corrections in the unperturbed Rashba-split subbands ${ }^{12}$ and, in combination with the Dresselhaus contribution, is responsible for the anisotropy in $\bar{\varepsilon}$ and for the corrections in the total spinsplitting anisotropy, given by the allowed different expectation values (or matrix elements) designated as \langle\rangle$_{s s^{\prime}}$. Note that the \pm sign above stands for spin up or down along the polarization direction of the perturbed eigenstates, which are given by

$$
\begin{aligned}
\left|\Psi_{ \pm}\right\rangle= & \frac{\left|H_{\uparrow \downarrow}\right|}{\sqrt{\left|H_{\uparrow \downarrow}\right|^{2}+\left(\varepsilon_{ \pm}-H_{\uparrow \uparrow}\right)^{2}}} \\
& \times\left(\begin{array}{c}
1 \\
H_{\uparrow \downarrow}^{*}\left(\varepsilon_{ \pm}-H_{\uparrow \uparrow}\right) /\left|H_{\uparrow \downarrow}\right|^{2}
\end{array}\right) .
\end{aligned}
$$

Note also that $\varepsilon_{ \pm}=\varepsilon_{ \pm}\left(k_{\|}, \vartheta\right)$, so that such spin polarization depends now on both the absolute value and the direction of $\vec{k}_{\|}$. In particular, for a fixed wave-vector direction, the spin polarization direction now changes with increasing $k_{\|}$.

From the above equations, one clearly sees that in general the heterojunction in-plane SO anisotropy cannot be described by a single parameter. It is easy to see that only in the infinitebarrier and small- $k_{\|}$limit does the splitting obtained above reduce exactly to the commonly used expression $\Delta_{s}\left(k_{\|}, \vartheta\right)=$ $2 k_{\|} \sqrt{\alpha^{2}+\beta^{2}-2 \alpha \beta \sin 2 \vartheta}$ where $\beta=\gamma\left\langle k_{z}^{2}\right\rangle,\left\langle k_{z}^{2}\right\rangle$ being the average momentum squared along the growth direction and $\gamma$ the $k^{3}$ bulk parameter for the well material [note that in this limit $\left.\left\langle\gamma(z) k_{z}^{2}\right\rangle_{\uparrow \uparrow}=\left\langle\gamma(z) k_{z}^{2}\right\rangle_{\uparrow \downarrow}=\left\langle\gamma(z) k_{z}^{2}\right\rangle_{\downarrow \downarrow}=\beta\right]$.

In the much studied two-dimensional electron gas (2DEG) formed in $\mathrm{AlGaAs} / \mathrm{GaAs}$ heterojunctions, such SO anisotropy is particularly evident. In Fig. 1, using a color scale, the obtained SO splitting for electrons in these structures (at the Fermi energy) is plotted as a function of the 2DEG electron density $n_{s}$ and of the $\vec{k}_{F}$ direction (low-temperature bulk parameters are used $\left.{ }^{17,18}\right)$. It is interesting to note that the $\vec{k}$ direction with maximum splitting varies with $n_{s}$. We note

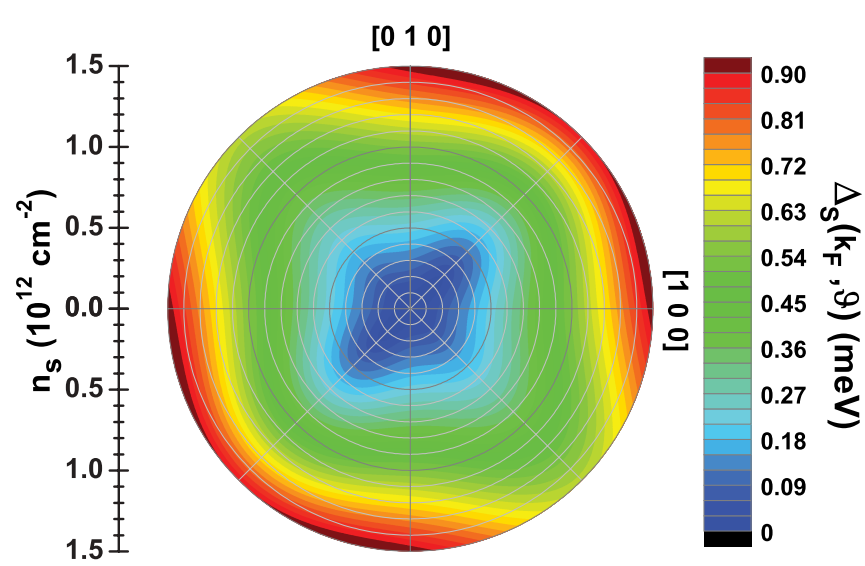

FIG. 1. (Color online) Total spin-orbit splitting (in color scale) at the Fermi level as a function of the 2DEG carrier concentration and Fermi wave-vector direction in an $\mathrm{Al}_{0.3} \mathrm{Ga}_{0.7} \mathrm{As} / \mathrm{GaAs}$ heterojunction. 
also that even for small $n_{s}$ (small $k_{\|}$) the SO splitting in these structures does not present the above mentioned twofold symmetry determined only by $\alpha / \beta$. This is due to the barrier penetration and can be understood by recalling that, when one reduces the barrier height at the interface and at the same time decreases $n_{s}$, the confinement and SIA are decreased so that $H_{D}$ (with fourfold symmetry) eventually dominates. Indeed, in the bulklike zero-barrier-height and very-small- $n_{s}$ limit, one has only the Dresselhaus SO term. For the same reason, even in narrow-gap-based heterojunctions $H_{D}$ should not be neglected (as is usually done) when barrier penetration is considerable (in this respect, see also the discussion below about $\alpha_{S d H}$ ).

Experimentally such anisotropy in GaAs heterojunctions has been most directly probed with the spin photocurrent ${ }^{9}$ (SPC) and ballistic electron spin resonance ${ }^{10}$ (BSR) techniques. Giglberger et al. ${ }^{9}$ interpreted their SPC data on the SO anisotropy with the $\alpha / \beta$ ratio and obtained values for this ratio equal to $7.6,2.8$, and 1.5 for the samples with $n_{s}$ equal to $1.1 \times 10^{11}, 1.3 \times 10^{11}$, and $1.8 \times 10^{11} \mathrm{~cm}^{-2}$, respectively. Instead, with BSR Frolov et al., ${ }^{10}$, also in an $n_{s}=1.1 \times$ $10^{11} \mathrm{~cm}^{-2} \mathrm{AlGaAs} / \mathrm{GaAs}$ heterojunction, measured $|\alpha-\beta|=$ $(2 \pm 0.5) \times 10^{-13} \mathrm{eV} \mathrm{m}$ and $|\alpha+\beta|=(0.6 \pm 0.2) \times 10^{-13}$ $\mathrm{eV} \mathrm{m}$, which correspond to $\alpha / \beta$ in the 1.4-3.2 range. There are thus still large experimental uncertainties with respect to the SO anisotropy. Nevertheless, we can compare our results with these measurements by taking our total splitting $\Delta_{s}$ for $\vec{k}_{F}$ along the two explored directions ([110] and [1 $\left.\left.\overline{10} 0\right]\right)$ and writing them as

$$
\Delta_{s}^{[110]}=|\alpha+\beta| k_{F}^{[110]} \quad \text { and } \quad \Delta_{s}^{[1 \overline{1} 0]}=|\alpha-\beta| k_{F}^{[1 \overline{1} 0]} .
$$

From these relations we obtain effective $\alpha / \beta$ ratios equal to $1.3,1.2$, and 1.1 for the same electron concentrations, i.e., for $n_{s}=1.1 \times 10^{11}, 1.3 \times 10^{11}$, and $1.8 \times 10^{11} \mathrm{~cm}^{-2}$ respectively; which reproduce well the observed $n_{s}$ dependence and are quantitatively much closer to the experimental data than other independent calculations of $\alpha$ and $\beta$ separately, in agreement with our conclusions with respect to the limitations of such $\alpha / \beta$ parametrization.

The SO splitting in 2DEGs is also often studied using analysis of the beating pattern in Shubnikov-de Haas $(\mathrm{SdH})$ oscillations, from which the electron density in the split subbands can be measured. The densities $n_{+}$and $n_{-}$(with $n_{+}+n_{-}=n_{s}$ ), in accord with the low-temperature occupation of each SO-split subband, can be easily calculated as

$$
n_{ \pm}=\frac{1}{4 \pi^{2}} \int_{0}^{2 \pi} \int_{0}^{\infty} \Theta\left[\varepsilon_{F}-\varepsilon_{ \pm}\left(k_{\|}, \vartheta\right)\right] k_{\|} d k_{\|} d \vartheta
$$

As is usually done in $\mathrm{SdH}$ data analysis, the difference $\delta n=$ $\left|n_{+}-n_{-}\right|$can then be used to obtain an effective Rashba coupling parameter $\alpha_{S d H}$ given by

$$
\alpha_{S d H}=\delta n \frac{\hbar^{2}}{m^{*}} \sqrt{\frac{\pi}{2\left(n_{s}-\delta n\right)}} .
$$

It is clear though that for the present III-V heterojunctions with SO anisotropy, this expression can give a measure of only the averaged splitting around the Fermi line, which will be an estimation of the usual Rashba coupling parameter $\alpha$ only in structures with negligible Dresselhaus contribution, as for example in insulator/InAs and insulator/InSb heterojunctions. With an InGaAs-based heterojunction $\left(n_{s}=1.4 \times\right.$ $10^{12} \mathrm{~cm}^{-2}$ ), Yang et al. ${ }^{19}$ observed a very clear beating pattern in the $\mathrm{SdH}$ oscillations corresponding to $\alpha_{S d H}=0.63 \times$ $10^{-11} \mathrm{eV}$ m. Using the dispersion relations in Eq. (9) we find, in reasonable agreement, $\alpha_{S d H}=0.55 \times 10^{-11} \mathrm{eV} \mathrm{m}$. If we neglect $H_{D}$, instead of 0.55 we get 0.72 , with a slightly larger deviation from the experimental value.

Another example is the InGaSb/InAlSb 2DEG spinsplitting data of Akabori et al., ${ }^{20}$ with a dominant Dresselhaus contribution in spite of its narrow gap, which can be understood with the barrier penetration effect discussed here. Finally, in agreement with our results there are also the recent data of Kohda et al. ${ }^{6}$ on the 2DEG persistent spin helix modes in InAlAs/InGaAs quantum wells (QWs) (with considerable barrier penetration) that do not fit with the $\alpha / \beta=1$ condition. However, it is clear that further measurements are still necessary in order to determine the SO anisotropy in III-V QWs. In particular, the observation and analysis of anomalous beating patterns in the magneto-oscillations have been very useful in similar studies in the bulk, ${ }^{21}$ and could be helpful in 2D systems as well, where they seem to have not been much explored yet.

In summary, we have seen that the present variational theory for the Rashba effect in semiconductor heterojunctions, with the Dresselhaus corrections included perturbatively, is able to give a good description of the spin-orbit anisotropy in III-V 2DEGs. The interpretation of the corresponding measurements has also been discussed, and the standard $\alpha / \beta$ parametrization criticized. Effects of the barrier penetration in the 2DEG SO splitting and anisotropy have been identified. The anisotropy was shown to be determined in general by more than one parameter, even in the small- $k$ limit (as opposed to the commonly used single $\alpha / \beta$ parameter). Reasonably good agreement with the available data indicates that the model calculation presented can be useful in the development of semiconductor spintronics.

The authors thank the Brazilian agencies CNPq, CAPES and FAPESB for financial support. E.A.A.S. is also grateful for the hospitality of the Scuola Normale Superiore di Pisa where part of this research was done.
${ }^{1}$ S. Datta and B. Das, Appl. Phys. Lett. 56, 665 (1990).

${ }^{2}$ I. Zutic, J. Fabian, and S. Das Sarma, Rev. Mod. Phys. 76, 323 (2004).

${ }^{3}$ H. C. Koo, J. H. Kwon, J. Eom, J. Chang, S. H. Han, and M. Johnson, Science 325, 1515 (2009).

${ }^{4}$ J. Wunderlich, B.-G. Park, A. C. Irvine, L. P. Zârbo, E. Rozkotová, P. Nemec, V. Novák, J. Sinova, and T. Jungwirth, Science 330, 6012 (2010).
${ }^{5}$ J. D. Koralek, C. P. Weber, J. Orenstein, B. A. Bernevig, S.-C. Zhang, S. Mack, and D. D. Awschalom, Nature (London) 458, 610 (2009).

${ }^{6}$ M. Kohda, V. Lechner, Y. Kunihashi, T. Dollinger, P. Olbrich, C. Schönhuber, I. Caspers, V. V. Bel'kov, L. E. Golub, D. Weiss, K. Richter, J. Nitta, and S. D. Ganichev, Phys. Rev. B 86, 081306(R) (2012).

${ }^{7}$ E. A. de Andrada e Silva, Phys. Rev. B 46, 1921(R) (1992). 
${ }^{8}$ R. Eppenga and M. F. H. Schuurmans, Phys. Rev. B 37, 10923(R) (1988).

${ }^{9}$ S. Giglberger, L. E. Golub, V. V. Bel'kov, S. N. Danilov, D. Schuh, C. Gerl, F. Rohlfing, J. Stahl, W. Wegscheider, D. Weiss, W. Prettl, and S. D. Ganichev, Phys. Rev. B 75, 035327 (2007).

${ }^{10}$ S. M. Frolov, S. Lüscher, W. Yu, Y. Ren, J. A. Folk, and W. Wegscheider, Nature (London) 458, 868 (2009); note however the different convention used for the crystallographic direction or parameter sign.

${ }^{11}$ M. A. Toloza Sandoval, A. Ferreira da Silva, E. A. de Andrada e Silva, and G. C. La Rocca, Phys. Rev. B 79, 241305(R) (2009).

${ }^{12}$ M. A. Toloza Sandoval, A. Ferreira da Silva, E. A. de Andrada e Silva, and G. C. La Rocca, Phys. Rev. B 83, 235315 (2011).

${ }^{13}$ The Rashba splitting is exactly linear in $k_{\|}$only in the infinite-barrier and parabolic approximation (i.e., neglecting the $\alpha$ energy dependence). However, in the GaAs/GaAlAs heterojunctions considered here, the deviation from the linear behavior is very small.
${ }^{14}$ G. Dresselhaus, Phys. Rev. 100, 580 (1955).

${ }^{15}$ M. Cardona, N. E. Christensen, and G. Fasol, Phys. Rev. B 38, 1806 (1988).

${ }^{16}$ E. A. de Andrada e Silva, G. C. La Rocca, and F. Bassani, Phys. Rev. B 50, 8523 (1994).

${ }^{17}$ Intrinsic Properties of Group IV Elements and III-V, II-VI and I-VII Compounds, edited by O. Madelung, Landolt-Börnstein, New Series, Group III, Vol. 22, Pt. a (Springer, Berlin, 1987).

${ }^{18}$ J.-M. Jancu, R. Scholz, E. A. de Andrada e Silva, and G. C. La Rocca, Phys. Rev. B 72, 193201 (2005).

${ }^{19}$ C. L. Yang, H. T. He, L. Ding, L. J. Cui, Y. P. Zeng, J. N. Wang, and W. K. Ge, Phys. Rev. Lett. 96, 186605 (2006).

${ }^{20}$ M. Akabori, V. A. Guzenko, T. Sato, Th. Schapers, T. Suzuki, and S. Yamada, Phys. Rev. B 77, 205320 (2008).

${ }^{21}$ See, for instance, G. C. La Rocca, M. M. Miller, and S. Rodriguez, Phys. Rev. B 40, 11723 (1989). 